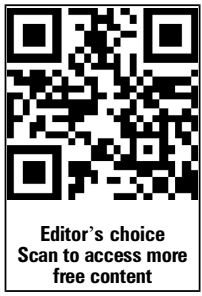

${ }^{1} \mathrm{PhD}$ Student, Department of Pharmacy, Health and Wellbeing, Faculty of Applied Sciences, University of Sunderland, Sunderland, UK ${ }^{2}$ Professor of General Practice and Primary Care, Department of Pharmacy, Health and Wellbeing, Faculty of Applied Sciences, University of Sunderland, Sunderland, UK ${ }^{3}$ Consultant in Community Gynaecology and Reproductive Healthcare/Head of Clinical Service, Department of Sexual Health, New Croft Centre, Newcastle upon Tyne Hospitals NHS Foundation Trust, Newcastle upon Tyne, UK ${ }^{4}$ Consultant General Surgeon with Bariatric Interest Department of General Surgery, Sunderland Royal Hospital, Sunderland, UK

\section{Correspondence to} Ms Yitka Graham, Faculty of Applied Sciences, Department of Pharmacy, Health and Wellbeing, University of Sunderland, Chester Road, Sunderland SR1 3SD, UK; yitka.graham@sunderland.ac.uk

Received 10 April 2014 Revised 17 July 2014

Accepted 27 July 2014

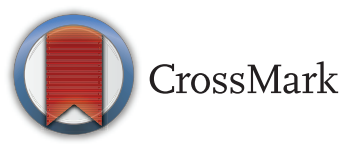

To cite: Graham Y, Wilkes $S$, Mansour D, et al. J Fam Plann Reprod Health Care 2014; $40: 241-244$

\title{
Contraceptive needs of women following bariatric surgery
}

\author{
Yitka Graham, ${ }^{1}$ Scott Wilkes, ${ }^{2}$ Diana Mansour, ${ }^{3}$ Peter K Small ${ }^{4}$
}

\section{INTRODUCTION}

With one in four adults classified as obese in the $\mathrm{UK},{ }^{1}$ bariatric surgery is becoming an increasingly common weight loss intervention. The number of procedures has increased from fewer than 1000 procedures performed in 2000 to almost 10000 in 2010. ${ }^{1}$ Compared to diet, exercise and pharmacotherapy, bariatric surgery offers sustained, long-term weight loss ${ }^{2}$ and improvement in obesity-related conditions. Following surgery, published data suggest that patients can expect up to a $72 \%$ chance of remission of type 2 diabetes, ${ }^{3}$ $46 \%$ resolution of hypertension ${ }^{4}$ and $75 \%$ improvement in sleep apnoea..$^{5}$ Weight loss through bariatric surgery has also been shown to improve menstrual cycle disorders and theoretically to improve fertility. ${ }^{6}$

The National Institute for Health and Care Excellence (NICE) guideline on management of adult obesity recommends bariatric surgery when other weight loss interventions have failed. ${ }^{8}$ National Health Service criteria for surgery are determined by a body mass index (BMI) (in $\mathrm{kg} / \mathrm{m}^{2}$ ), greater than 40 , or 35 with significant disease that could be improved with weight loss, such as type 2 diabetes or hypertension. For severely obese patients with a BMI $>50$, bariatric surgery may be considered as a first-line option. ${ }^{8}$

The UK National Bariatric Surgery Registry (NBRS) shows that $80 \%$ of bariatric procedures were performed in women, with the majority being in the reproductive age group. ${ }^{1}$ Compared to women with a normal BMI, obese women may be up to three times more likely to experience infertility. ${ }^{7}$ Generally, women who undergo bariatric surgical procedures experience improved fertility and maternal outcomes compared to obese cohorts. ${ }^{67}$ There is a paucity of literature investigating the effects of the different bariatric procedures on contraceptive efficacy, and whether specific methods should be recommended. Current recommendations advise against pregnancy following bariatric surgery for at least 1 year afterwards, ${ }^{7}{ }^{9}$ as the first 2 years following surgery are the period of most rapid weight loss. ${ }^{2}$ In addition, the surgically altered digestive physiology means that patients have to adjust to new eating habits and there is potential for nutrient deficiencies to occur; ${ }^{10}$ this risk is increased in patients who undergo malabsorptive procedures. The effects of this on maternal and fetal outcomes have not been accurately determined. If present rates of obesity and bariatric surgery continue to rise, an emerging 'subgroup' of women will require more specialist contraceptive advice with reproductive health decisions dependent on the procedure performed, weight loss experienced and possible effects on future pregnancies.

\section{BARIATRIC SURGICAL PROCEDURES}

Bariatric procedures exhibit their effect on weight loss and comorbidity improvement through either a restrictive or a malabsorption mechanism, or a combination of both. The most common procedure performed in the UK is the gastric bypass (55\%), ${ }^{1}$ with gastric sleeve and gastric banding performed less frequently. Most procedures are performed laparoscopically.

Gastric bypass surgery creates a small pouch that is separated from the rest of the stomach (Figure 1). The small intestine is divided in the middle of the jejunum into two limbs. The lower limb is attached via an anastomosis to the gastric pouch and the upper limb is attached further down the jejunum, bypassing the stomach, duodenum and proximal jejunum. The stomach pouch is only able to hold a small amount of food (restrictive); malabsorption occurs as a result of bypassing the proximal small bowel. The malabsorptive effect of gastric bypass requires lifelong supplementation to prevent nutrient deficiencies. $^{11}$ Levels of fasting glucose, insulin, ghrelin (a hormone primarily responsible for appetite stimulation) and the gut hormone peptide-YY (an appetite regulant) are significantly altered following 


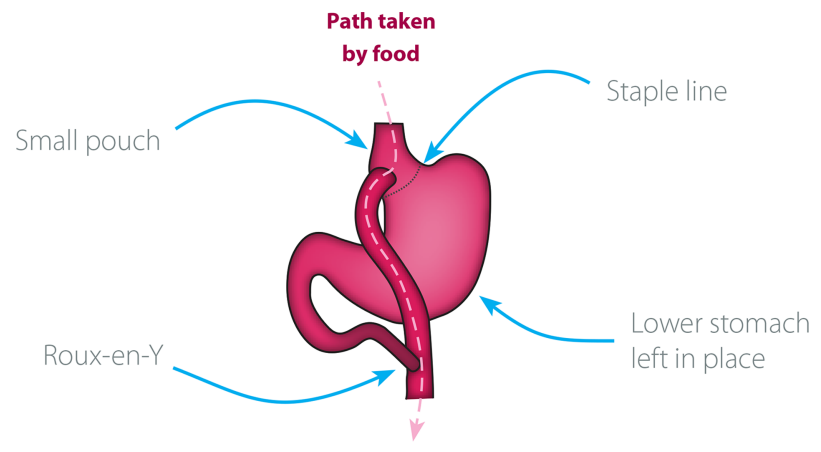

Figure 1 Diagrammatic representation of a Roux-en-Y gastric bypass procedure. Image reproduced with the kind permission of Dendrite Clinical Systems Ltd.

bariatric surgery and depend on the type of procedure performed. ${ }^{12} 13$ The gastric sleeve is a restrictive procedure that involves removing $80 \%$ of the stomach, leaving a sleeve-shaped organ (Figure 2). There is a theoretical impact on appetite suppression through reduced production of ghrelin, but its metabolic mechanisms are still not fully understood. ${ }^{14}$ It is proposed that the effect of the gastric sleeve on the absorption of oral hormones cannot be accurately determined at the present time, and this is an identified area for future research. Gastric banding involves placing an inflatable band around the upper portion of the stomach, which creates a small pouch above the site of the band, allowing for the size of the stomach opening to be adjusted by filling the band with a saline solution through a subdermal port. Gastric banding is reversible, and wholly restrictive with no malabsorptive component. The number of gastric banding procedures performed in the UK is declining due in part to high rates of removal and revision surgery attributed to patient intolerance, slippage, reflux and infection. ${ }^{15}$

\section{REPRODUCTIVE HEALTH AND THE BARIATRIC PATIENT}

Fertility is negatively affected by obesity, ${ }^{16}$ but the rapid weight loss imposed by bariatric surgery means women will experience improved fertility, ${ }^{17}$ and be at risk of an unplanned pregnancy. There is no universal consensus on the timeframe, but the current advice to avoid pregnancy for at least 1 year, and possibly up to 2 years after surgery, ${ }^{79}$ is intended to reduce any theoretical risks to maternal and fetal health as a result of nutrient deficiencies and rapid weight loss.

Adverse maternal events may be lower in bariatric surgical patients compared with their obese counterparts. A retrospective study comparing 346 pregnant women who delivered before bariatric surgery with 354 women who delivered after bariatric surgery found a lower incidence of gestational diabetes $(27 \%$ vs $8 \%) .{ }^{18} \mathrm{~A}$ systematic review that included three matched case-controlled studies by Maggard et al., ${ }^{7}$ comparing women who had undergone bariatric surgery with obese controls, reported a reduced risk

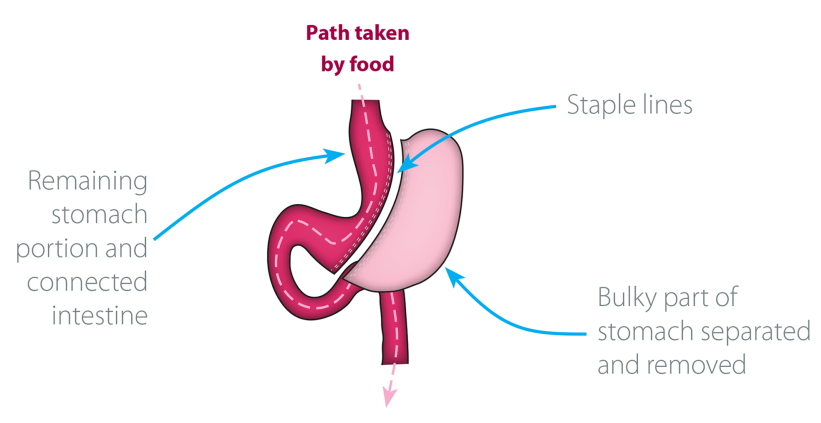

Figure 2 Diagrammatic representation of a sleeve gastrectomy procedure. Image reproduced with the kind permission of Dendrite Clinical Systems Ltd.

of developing pregnancy-induced hypertension and pre-eclampsia, along with lower rates of gestational diabetes. In addition, some studies suggest fewer neonatal complications such as premature delivery, macrosomia and low birth weight following bariatric surgery compared to obese controls. ${ }^{79}$

Thus the choice of contraceptive method in bariatric patients requires careful consideration to ensure that it is effective, acceptable to the woman, and that pregnancy is avoided.

\section{ABSORPTION OF ORAL CONTRACEPTIVES FOLLOWING BARIATRIC SURGERY}

Adaptation to post-surgical life includes changes to eating habits that may result in vomiting and diarrhoea, known causes of breakthrough bleeding, and reduced efficacy in women taking all types of oral contraceptives. Vomiting and diarrhoea may occur with all procedures, and are generally a result of poor food choices. ${ }^{19}$ Other causes of vomiting include food becoming stuck (banding and sleeve) and anastomotic stenosis, narrowing of the new connection between the stomach and lower intestine (gastric bypass). Frequent or prolonged vomiting should always be investigated.

The pharmacokinetics of oral contraception is affected by the type of procedure performed. Oral estrogen is mostly absorbed in the upper gastrointestinal tract, which is reshaped as a result of bariatric surgery, particularly with gastric bypass as this altered digestive physiology results in malabsorption. The United States Medical Eligibility Criteria for Contraceptive Use advises against oral contraceptives in bariatric patients. ${ }^{20}$ A 'before and after' comparison study of the pharmacokinetics of an ethinylestradiol/levonorgestrel contraceptive pill 1 month before and 4 months post-gastric bypass is currently recruiting surgery-seeking females aged between 18 and 40 years. The primary outcome measure is twice-weekly serum hormone levels before and after surgery; the secondary outcome measures include twice-weekly endometrial thickness measured by transvaginal ultrasound for the duration of the study. ${ }^{21}$ 
Table 1 Proposed contraception for patients following bariatric surgery

\begin{tabular}{llllllll}
\hline $\begin{array}{l}\text { Bariatric } \\
\text { procedure }\end{array}$ & COCs & POPs & $\begin{array}{l}\text { DMPA or } \\
\text { NET-EN }\end{array}$ & $\begin{array}{l}\text { Progestogen-only } \\
\text { implant }\end{array}$ & Cu-IUD & LNG-IUS & $\begin{array}{l}\text { Combined hormonal } \\
\text { patch, vaginal ring }\end{array}$ \\
\hline Gastric bypass & 3 & 3 & 2 & 2 & 1 & 1 & 3 \\
Gastric sleeve & 2 & 2 & 2 & 2 & 1 & 1 & 2 \\
Gastric banding & 2 & 2 & 2 & 2 & 1 & 1 & 2 \\
\hline
\end{tabular}

Definition of categories is based on UK Medical Eligibility Criteria for Contraceptive Use (UKMEC) ${ }^{23}$ as follows:

$1 \mathrm{~A}$ condition for which there is no restriction for the use of the contraceptive method.

2 A condition where the advantages of using the method generally outweigh the theoretical or proven risks.

3 A condition where the theoretical or proven risks usually outweigh the advantages of using the method. The provision of a method requires expert clinical judgement and/or referral to a specialist contraceptive provider, since use of the method is not usually recommended unless other more appropriate methods are not available or not acceptable.

$4 \mathrm{~A}$ condition which represents an unacceptable health risk if the contraceptive method is used.

COCS, combined oral contraceptives; Cu-IUD, copper-containing intrauterine device; DMPA; depot medroxyprogesterone acetate; LNG-IUS, levonorgestrelreleasing intrauterine system; NET-EN, norethisterone enantate; POPs, progestogen-only pills.

Currently it is thought that the efficacy of progestogen-only pills and emergency contraception pills following malabsorptive procedures may be reduced and are not recommended. ${ }^{20}$

No evidence was found to suggest a reduction in the effectiveness of oral hormones following restrictive procedures. ${ }^{20}$

\section{NON-ORAL CONTRACEPTIVE USE FOLLOWING BARIATRIC SURGERY}

Patients experience significant weight loss after surgery, resulting in loose skin, which may affect contraceptive choices (e.g. there is anecdotal evidence to suggest difficulties in finding a suitable site for applying transdermal patches and making insertion of subdermal implants technically more difficult).

A study of three case reports of obese women (preoperative BMI range $51.8-64.7 \mathrm{~kg} / \mathrm{m}^{2}$ ) with etonogestrel implants inserted prior to gastric bypass showed that levels of etonogestrel decreased with weight loss, but these levels remained sufficiently high to provide contraceptive efficacy. ${ }^{22}$

It is thought that the association of weight gain with injectable methods ${ }^{23}$ may be unacceptable to bariatric patients. In addition, the existing potential for bone mineral density loss with injectable methods ${ }^{23}$ that may also occur with bariatric procedures ${ }^{17}$ is still under investigation, so this method should be regarded with caution at present.

Obese women may find vaginal rings, female condoms, diaphragms and cervical caps difficult to insert and intrauterine contraceptive threads difficult to check. Guidance for diaphragm users recommends visiting a health care professional to check the correct sizing of this barrier method following a weight change of $3 \mathrm{~kg},{ }^{23}$ making this an impractical choice for bariatric patients.

The psychosocial impact of bariatric surgery is known to affect interpersonal relationships; ${ }^{24}$ irreversible methods of contraception such as male and female sterilisation or non-surgical blockage of the Fallopian tubes $\left(\right.$ Essure $\left.^{\circledR}\right)$, leading to permanent lifestyle changes, should be carefully considered following bariatric surgery to avoid later regret.

\section{SUGGESTED CONTRACEPTIVE METHODS FOR BARIATRIC PATIENTS}

Based on current UK and USA contraceptive guidelines $^{20}{ }^{23}$ for obese women, and accounting for the theoretical risk for bariatric procedures to reduce oral hormone absorption, intrauterine devices (IUDs) (either hormonal or copper) should be offered as a first-line contraceptive method for bariatric patients. IUDs offer long-term reversible contraception that is not affected by surgically imposed weight loss or malabsorption, but the device threads may be difficult to check until significant weight loss is achieved. Current UK contraception recommendations for obese women do not assess the impact of bariatric procedures. ${ }^{23}$ Table 1 is a proposed guide to contraception recommendations for obese women, based on the format of the UK Medical Eligibility Criteria for Contraceptive $\mathrm{Use}^{23}$ and assumes a postoperative BMI of at least $35 \mathrm{~kg} / \mathrm{m}^{2}$, based on NICE minimum weight eligibility criteria for bariatric surgery.

Male condoms are also an option; but as a patient's weight decreases, the range of contraceptive options increases. The risk of adverse events associated with specific contraceptive methods, obesity, pregnancy and surgical procedure must be balanced against the needs of the individual bariatric patient.

\section{CONCLUSIONS}

Obese women undergoing bariatric surgery are an emerging contraceptive-seeking cohort whose needs are different to those of obese women. Rapid weight loss and nutritional considerations mean that pregnancy should be avoided for at least 1 year following surgery. Patients undergoing procedures resulting in malabsorption should not be offered oral hormonal contraception until the pharmacokinetic changes associated with this form of surgery are more clearly understood. Future research should focus on 
contraceptive outcomes in this cohort and currently performed procedures.

\section{Competing interests None.}

Provenance and peer review Not commissioned; externally peer reviewed.

Editor's note Readers may also be interested in a very recent (August 2014) BMJ State of the Art Review entitled 'Bariatric surgery for obesity and metabolic conditions in adults' (Arterburn DE, Courcoulas AP, BMJ 2014;349:g3961). This excellent review summarises recent evidence relating to the safety, efficacy and metabolic outcomes of bariatric surgery to guide clinical decision making, and complements the specific issues discussed by Graham et al. in this commentary.

\section{REFERENCES}

1 Welbourn R, Fiennes A, Walton P, et al. The National Bariatric Surgery Registry: First Registry Report to March 2010.

Henley-on-Thames, UK: Dendrite Clinical Systems Limited, 2011.

2 Sjöström L, Narbro K, Sjöström CD, et al. Effects of bariatric surgery on mortality in Swedish obese subjects. N Engl J Med 2007;357:741-752.

3 Sjöström L. Review of the key results from the Swedish Obese Subjects (SOS) trial - a prospective controlled intervention study of bariatric surgery. J Intern Med 2013;273:219-234.

4 Mingrone G, Panunzi S, de Gaetano A, et al. Bariatric surgery versus conventional medical therapy for type 2 diabetes. N Engl J Med 2012;366:1577-1585.

5 Kaska L, Kobiela J, Abacjew-Chmylko A, et al. Nutrition and pregnancy after bariatric surgery. ISRN Obes 2013;2013:492060.

6 Guelinckx I, Devlieger R, Vansant G. Reproductive outcome after bariatric surgery: a critical review. Hum Reprod Update 2009;15:189-201.

7 Maggard MA, Yermilov I, Li Z, et al. Pregnancy and fertility following bariatric surgery: a systematic review. JAMA 2008;300:2286-2296.

8 National Institute for Clinical Excellence (NICE). Obesity: Guidance on the Prevention, Assessment and Management of Overweight and Obesity in Adults and Children. London: NICE, 2006.

9 American College of Obstetricians and Gynecologists (ACOG). ACOG practice bulletin: bariatric surgery and pregnancy 105 . Obstet Gynecol 2009;113:1405-1413.

10 Mechanick J, Youdim A, Jones D, et al. Clinical practice guidelines for the perioperative nutritional, metabolic, and nonsurgical support of the bariatric surgery patient - 2013 update: cosponsored by American Association of Clinical
Endocrinologists, the Obesity Society, and American Society for Metabolic and Bariatric Surgery. Endocr Pract 2013;19:337-372.

11 Kjaer MM, Nilas L. Pregnancy after bariatric surgery - a review of benefits and risks. Acta Obstet Gynecol Scand 2013;92:264-271.

12 Jacobsen S, Olesen S, Dirksen C, et al. Changes in gastrointestinal hormone responses, insulin sensitivity and beta-cell function within 2 weeks of gastric bypass in non-diabetic subjects. Obes Surg 2012;22:1084-1096.

13 Eerdekens A, Debeer A, Van Hoey G, et al. Maternal bariatric surgery: adverse outcomes in neonates. Eur J Pediatr 2010;169:191-196.

14 American Society for Metabolic and Bariatric Surgery (ASMBS). Updated Position Statement on Sleeve Gastrectomy as a Bariatric Procedure. Gainesville, FL: ASMBS, 2011.

15 Brown J, Boyle M, Mahawar K, et al. Laparoscopic adjustable gastric band survival in a high-volume bariatric unit. Br J Surg 2013;100:1614-1618.

16 Murthy AS. Obesity and contraception: emerging issues. Semin Reprod Med 2010;28:156-163.

17 Paulen M, Zapata L, Cansino C, et al. Contraceptive use among women with a history of bariatric surgery. Contraception 2010;82:86-94.

18 Burke A, Bennett W, Jamshidi R, et al. Reduced incidence of gestational diabetes with bariatric surgery. J Am Coll Surg 2010;211:169-175.

19 Pelizzo G, Calcaterra V, Fusillo M, et al. Malnutrition in pregnancy following bariatric surgery: three clinical cases of fetal neural defects. Nutr J 2014;13:59.

20 Centers for Disease Control and Prevention (CDC). United States Medical Eligibility Criteria for Contraceptive Use. Atlanta, GA: CDC, 2010.

21 National Institutes of Health. Changes in Oral Contraceptive Hormones Following Bariatric Surgery. 2013. http:// clinicaltrials.gov/show/NCT01941134 [accessed 19 August 2014].

22 Ciangura C, Corigliano N, Basdevant A, et al. Etonogestrel concentrations in morbidly obese women following Roux-en-Y gastric bypass surgery: three case reports. Contraception 2011;84:649-651.

23 Faculty of Sexual \& Reproductive Healthcare (FSRH). UK Medical Eligibility Criteria for Contraceptive Use (UKMEC 2009). London, UK: FSRH, 2009.

24 Bocchieri L, Meana M, Fisher B. Perceived psychosocial outcomes of gastric bypass surgery: a qualitative study. Obes Surg 2002;12:781-788. 